\title{
Le brevet, un « couteau suisse » pour inventer et innover
}

\section{The Patent, a "Swiss Knife" to invent and to innovate}

\author{
Yann de Kermadec ${ }^{1}$ \\ 1 Julhiet-Sterwen, Paris, France, y.de-kermadec@julhiet-sterwen.com
}

\begin{abstract}
RÉSUMÉ. Le langage des brevets est au cœur des interactions entre l'innovation, les inventions et les brevets. Pour rédiger un brevet, il faut répondre à une question logique, puissante mais pas naturelle pour les innovateurs : « Quelles combinaisons nouvelles de moyens proposez-vous ? ». Si l'on traduit les phrases compliquées et souvent très longues des revendications des brevets sous la forme de schémas, appelés « arbres des moyens », le " langage des brevets » devient très clair pour les concepteurs. L'arbre des moyens facilite l'utilisation des brevets dans une démarche globale appelée " Innover grâce aux brevets ». Etant à la fois un outil stratégique pour protéger et exploiter les innovations, une mine d'informations et un langage de conception puissant, les brevets permettent de dynamiser, sécuriser et mieux exploiter les projets d'innovation. ABSTRACT. Patent language is located at the center of interactions between innovation, inventions and patents. To elaborate a patent, one must answer a logical, powerful but non-natural question, "Which new combinations of means do you propose? ». If you translate very long and complicated phrases of patent claims into drawings called "means tree", the patent language becomes very clear to engineers. The means tree facilitates the use of patents within a global approach named « Innovate thanks to patents ». Being simultaneously a strategic tool to protect and exploit innovations, a mine of information and a powerful design language, allow to foster, to secure and to better exploit innovation projects.

MOTS-CLÉS. Innovation, invention, brevet, créativité, management des connaissances, arbre des moyens.

KEYWORDS. Innovation, invention, patent, creativity, Knowledge management, means tree.
\end{abstract}

\section{Introduction}

Le système des brevets a été créé il y a plus de deux siècles pour accélérer le progrès technique, grâce à une reconnaissance pertinente des créateurs et un bon partage des connaissances. De la plume d'oie à internet, le monde a beaucoup évolué ! Cependant, le principe fondateur des brevets est plus que jamais d'actualité.

Par ailleurs, avec la taylorisation des activités, les brevets sont principalement devenus une affaire de spécialistes. La plupart des innovateurs connaissent mal les brevets.

Mais depuis quelques mois, grâce à une représentation schématique des revendications des brevets, appelée « arbre des moyens », tous les innovateurs peuvent comprendre les brevets dans leur domaine technique. Cela ouvre des perspectives nouvelles que cet article commence à défricher.

Après avoir défini quelques termes clés, cet article présente, de manière générale, les relations qui existent entre l'innovation, les inventions et les brevets (deuxième partie de l'article).

La troisième partie présente l'arbre des moyens et ses utilisations qui permettent de dynamiser toutes les étapes de l'innovation.

La quatrième partie explique comment le système des brevets peut servir de modèle pour le management des connaissances pour l'innovation. 


\section{Invention, innovation, propriété intellectuelle : quelques repères}

\subsection{Quelques définitions}

« Je ne discute pas d'un mot tant qu'on ne définit pas le mot» (Blaise Pascal)

L'innovation est un « vaste sujet». Cette activité complexe intègre de nombreuses dimensions :

- Des dimensions classiques : stratégie, marketing, technologie, finance, juridique, management...

- Des dimensions plus spécifiques : créativité, propriété intellectuelle, psychologie des créateurs...

C'est la raison pour laquelle les termes utilisés pour l'innovation ont parfois des sens relativement différents en fonction des acteurs, très variés, qui utilisent ces termes. Certains parlent même d'une « belle pagaïe sémantique », qui perturbe fortement les coopérations entre ces acteurs !

Il paraît donc important de définir les termes clés de l'innovation, qui seront utilisés dans cet article. La première définition à fixer est celle du terme « innovation » ou plutôt, pour privilégier l'action, le verbe « innover » : Innover, c'est introduire, dans un domaine, quelque chose de nouveau (Y. d. Kermadec 2001), (Y. d. Kermadec 2009). Cette définition est très proche de celle donnée par les dictionnaires classiques, par exemple « introduire du nouveau dans un système établi » (Collectif-Robert 2012) .

La définition retenue présente plusieurs avantages :

- Elle est utilisable par tous et pour toutes les innovations, à la fois pour la vie professionnelle et pour la vie personnelle;

- Elle synthétise la « méthode » qu'il faut utiliser pour innover ;

- Elle est totalement cohérente avec les définitions d'autres termes (domaine, découverte, idée, création, invention...) utilisés pour l'innovation et pour la propriété intellectuelle.

\subsubsection{De l'idée à l'innovation...}

Une idée, c'est une proposition d'innovation. Cette idée débouche sur une création technique lorsqu'elle est suffisamment formalisée pour que des personnes « du métier » soient capables de la concrétiser. Si cette création technique est une "première mondiale», c'est une invention... qui contribuera peut-être au développement d'une ou plusieurs innovations.

Les créations techniques et les innovations se nourrissent d'idées mais aussi, et surtout, de nombreuses inventions, plus ou moins anciennes (la roue, la vis, le moulage, la batterie, le moteur électrique, le téflon, le GPS, internet...).

Au cour de la création, il y a la conception, l'action de concevoir. Concevoir (quelque chose), c'est faire la création intellectuelle (de cette chose). Cette définition met en évidence le lien très fort qui existe entre la conception et la propriété intellectuelle.

\subsubsection{Utiliser la propriété intellectuelle... pour innover}

Le brevet (d'invention) est un titre de propriété intellectuelle qui protège les inventions techniques.

La propriété intellectuelle (PI), c'est tout ce qui concerne la propriété des créations intellectuelles (droit de la PI, droits de PI d'une entreprise, acteurs de la PI, management de la PI dans une entreprise, économie de la PI...).

Le management de la PI dans l'entreprise, c'est le management de l'ensemble des aspects PI qui sont intégrés dans les fonctions et les activités de l'entreprise (veille PI, contrats pour l'innovation collaborative, études de liberté d'exploitation, créativité brevets, acquisition et exploitation des droits de PI, litiges PI, fiscalité PI, compétences PI...) (Breesé 2002). 


\subsection{Relations entre l'innovation, les inventions et les brevets}

L'innovation technique se nourrit des inventions et des brevets. La Figure 1 permet de décrire les relations très riches qui existent entre ces trois domaines et, de manière plus générale, entre l'innovation, les créations techniques et la propriété intellectuelle.

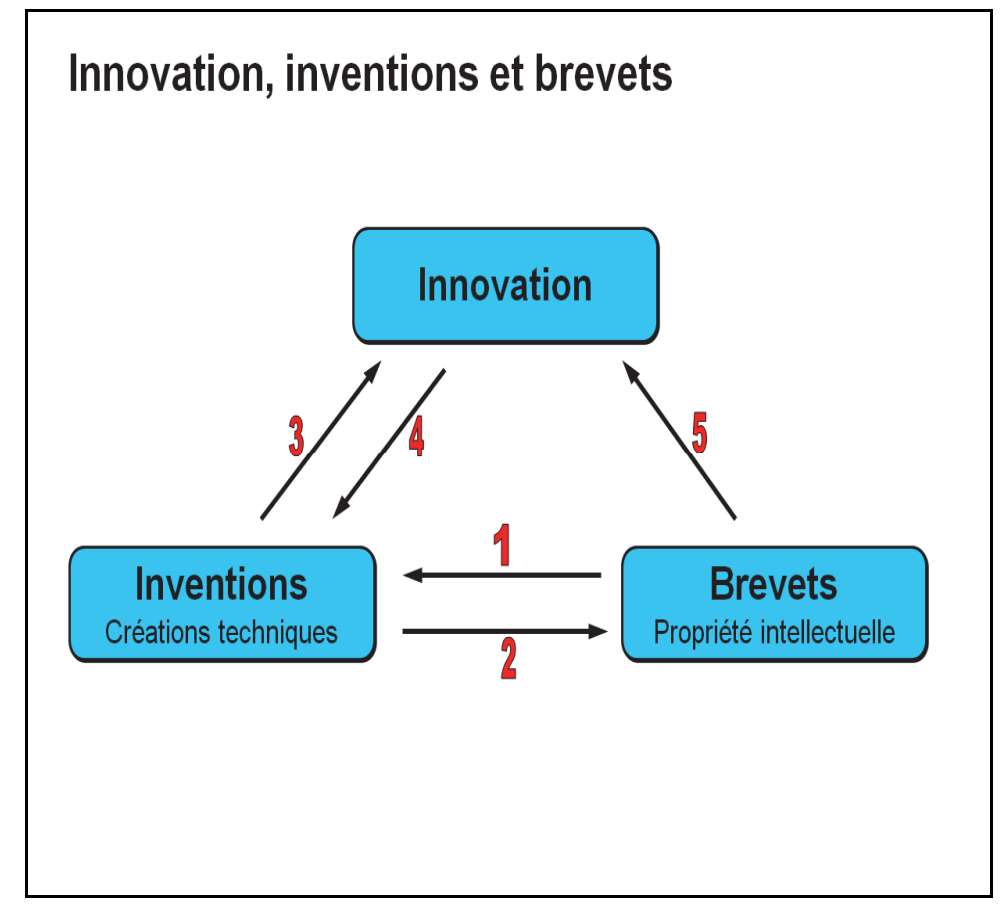

Figure 1. Innovation, inventions et brevets - Source : auteur

\subsubsection{Relation « Brevets => Inventions »}

Les brevets existants sont très utiles pour faire des créations techniques. En effet, toutes les solutions techniques que l'on crée aujourd'hui ne font que combiner de manière nouvelle, dans un domaine, des moyens que l'on trouve gratuitement dans la base mondiale des brevets ! Cela paraît étonnant mais, pour l'instant, l'auteur n'a pas encore trouvé d'exception à cette « règle ». L'auteur remercie à l'avance tout lecteur qui en trouverait à les lui transmettre.

Ces créations techniques sont des inventions si elles sont nouvelles, au niveau mondial.

\subsubsection{Relation «Inventions $=>$ Brevets »}

Les inventions sont brevetables, si elles satisfont aux trois critères de brevetabilité définis dans le Code de la propriété intellectuelle : nouveauté - activité inventive - application industrielle.

Pour les concepteurs, le seul critère vraiment important est le critère «nouveauté ». Le critère « application industrielle » est toujours validé, sauf dans des cas très particuliers (méthodes d'affaires...). Quant au critère « activité inventive », c'est un critère assez subjectif. Seul un spécialiste des brevets est capable de bien l'appréhender et de rédiger le brevet " pour faire apparaître une belle activité inventive » (selon l'expression consacrée des spécialistes) : c'est l'art de la plaidoirie !

Les brevets permettent de protéger les inventions. De manière plus large, la propriété intellectuelle permet de protéger la plupart des créations techniques sous des formes variées (brevet, publication, secret, contrats...).

\subsubsection{Relation «Inventions => Innovation »}

Les inventions nourrissent directement les innovations techniques et, indirectement, les autres types d'innovations. 
L'invention de la souris d'ordinateur l'illustre bien. Cette célèbre « souris » a permis à un très large public d'utiliser un ordinateur... ce qui a indirectement contribué au développement de nombreuses innovations dans les services : accès à des bases de données, messagerie électronique, e-commerce...

\subsubsection{Relation «Innovation => Inventions »}

Les innovations, pendant leur développement et leur exploitation, génèrent de nouveaux besoins et de nouvelles opportunités. C'est la principale source pour faire des inventions.

Voici un exemple de la vie courante. Vers 1815 un Allemand, Karl von Drais, invente l'ancêtre de la bicyclette, la draisienne. L'utilisateur est assis sur une selle et ses pieds sont en appui au sol pour se propulser... et freiner! Le vélocipède, puis la bicyclette, ont fait tomber dans l'oubli la draisienne... qui est réapparue il y a quelques années pour les enfants qui apprennent à faire de la bicyclette... ce qui a donné l'idée à un inventeur de déposer un brevet pour une draisienne-trottinette. En faisant pivoter de $180^{\circ}$ une partie du cadre, la draisienne devient trottinette et inversement (brevet $\mathrm{n}^{\circ}$ WO 2013/090953 A1).

\subsubsection{Relation « Brevets => Innovation »}

Les brevets, quand ils sont bien utilisés, facilitent les coopérations et l'exploitation paisible et fructueuse des innovations.

Quand on ne sait pas qui est propriétaire de quoi, il n'y a pas de coopération possible. PSA, par exemple, reçoit chaque année plus de 1000 « inventions non sollicitées ». Ce sont des personnes extérieures à PSA qui lui proposent des innovations dans le domaine de l'automobile. PSA pose immédiatement une question aux personnes qui proposent ces innovations : «Avez-vous déposé un brevet?». «Si c'est le cas, nous pouvons regarder ce dont vous êtes potentiellement propriétaire et voir si cela nous intéresse. Si ce n'est pas le cas, nous ne voulons pas connaître ce que vous proposez et en discuter. Il y a de fortes chances que nous nous disputions et que vous nous accusiez de vous avoir volé vos idées! ».

Ces explications sur les relations qui existent entre l'innovation, les inventions et les brevets mettent bien en évidence le fait que la puissance de l'innovation repose principalement sur la qualité des interactions entre les créations techniques et les brevets.

Le langage des brevets ${ }^{1}$ est au cœur de ces interactions. Ce langage est logique et puissant mais il n'est pas naturel pour les concepteurs et, de manière plus large, pour les innovateurs... d'où l'intérêt de «l'arbre des moyens », présenté ci-dessous, qui rend le langage des brevets accessible à tous.

\section{L'arbre des moyens : un langage pour innover et bien utiliser les brevets}

\subsection{Le « langage des brevets » est-il accessible à tous?}

Le système des brevets, créé il y a plus de deux siècles, repose sur un principe remarquable.

Un brevet est un «contrat» entre un inventeur (le plus souvent une entreprise) et la Collectivité. L'inventeur explique comment on peut réaliser son invention. En échange de cette information précieuse, l'inventeur peut obtenir un monopole pour l'exploitation de son invention, pendant 20 ans au maximum.

Pour que ce contrat soit pleinement « gagnant-gagnant», il faut que les concepteurs soient capables de lire « vite et bien » les brevets. Mais, actuellement, relativement peu de concepteurs et d'innovateurs ont cette compétence.

Les concepteurs et les innovateurs sont-ils incapables de comprendre le « langage des brevets »? 


\subsection{L’arbre des moyens: le « langage des brevets " traduit en schémas}

Les textes des premiers brevets étaient des descriptions techniques classiques. Les concepteurs lisaient donc facilement les brevets.

$\mathrm{Au}$ fil du temps, les offices de brevets ont imposé des règles de rédaction qui permettent de mieux répondre à deux questions juridiques clés, qui sont liées :

- «Quelles sont les limites de ce qui est protégé par ce brevet?»;

$-«$ Cette solution technique contrefait-elle ce brevet?».

Progressivement, les pays ont adopté l'utilisation des revendications dans les textes des brevets. Les revendications permettent d'aller au cœur d'une solution technique car elles posent aux inventeurs potentiels une question clé : «Quelles sont les combinaisons nouvelles de moyens proposées ?».

Les spécialistes des brevets ont ainsi inventé un langage de protection remarquable... qui est également un langage de conception puissant. Concevoir, en effet, consiste à combiner des moyens pour répondre à un besoin.

Mais les concepteurs ont beaucoup de mal à comprendre les revendications, qui sont des phrases compliquées et souvent très longues.

En revanche, si l'on traduit les revendications sous la forme de schémas, appelés « arbres des moyens », le « langage des brevets » devient très clair pour les concepteurs.

Voici, par exemple, un arbre des moyens qui correspond à la première revendication du brevet FR 2 752 374, dont le titre est « Dispositif releveur de produits, notamment de condiments, disposés dans un récipient ».

Les dessins de ce brevet, sur la Figure 2, présentent un dispositif releveur seul (Fig 3a) et un dispositif releveur dans un bocal (Fig 4a).

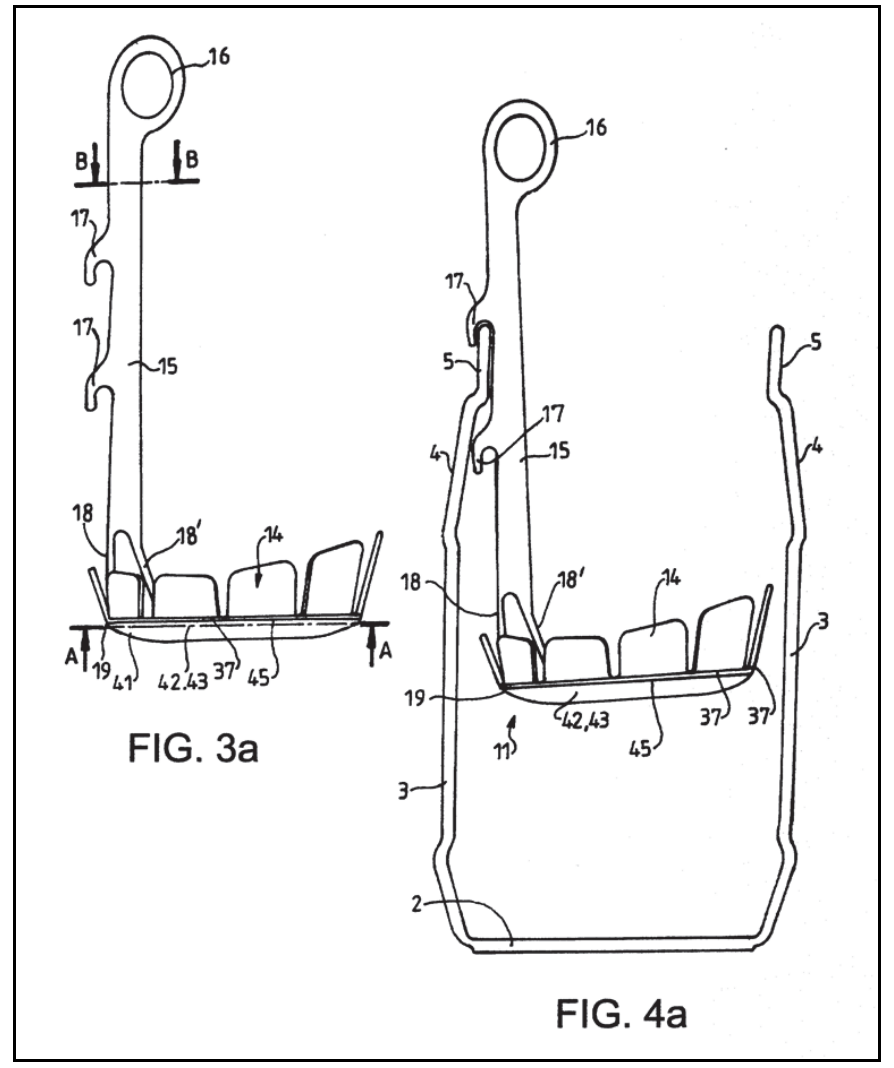

Figure 2. Dessins du brevet FR 2752374 - Source : brevet FR 2752374 
La Figure 3 présente la revendication 1 du brevet FR 2752374 : une phrase de quinze lignes, difficile à comprendre pour les non-spécialistes !

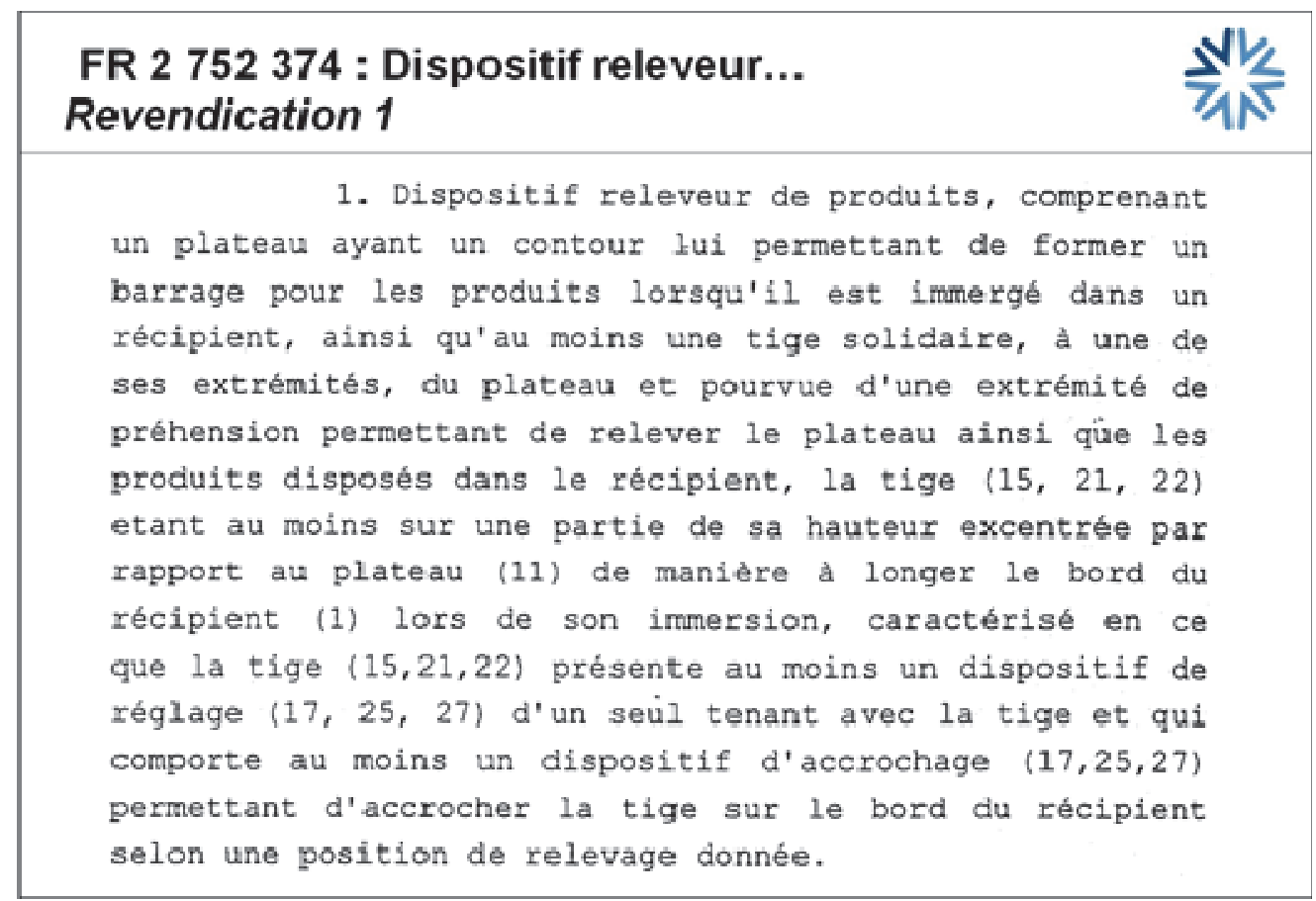

Figure 3. Revendication 1 du brevet FR 2752374 - Source : brevet FR 2752374

La Figure 4 présente, sous la forme d'un arbre des moyens, la revendication présentée à la Figure 3.

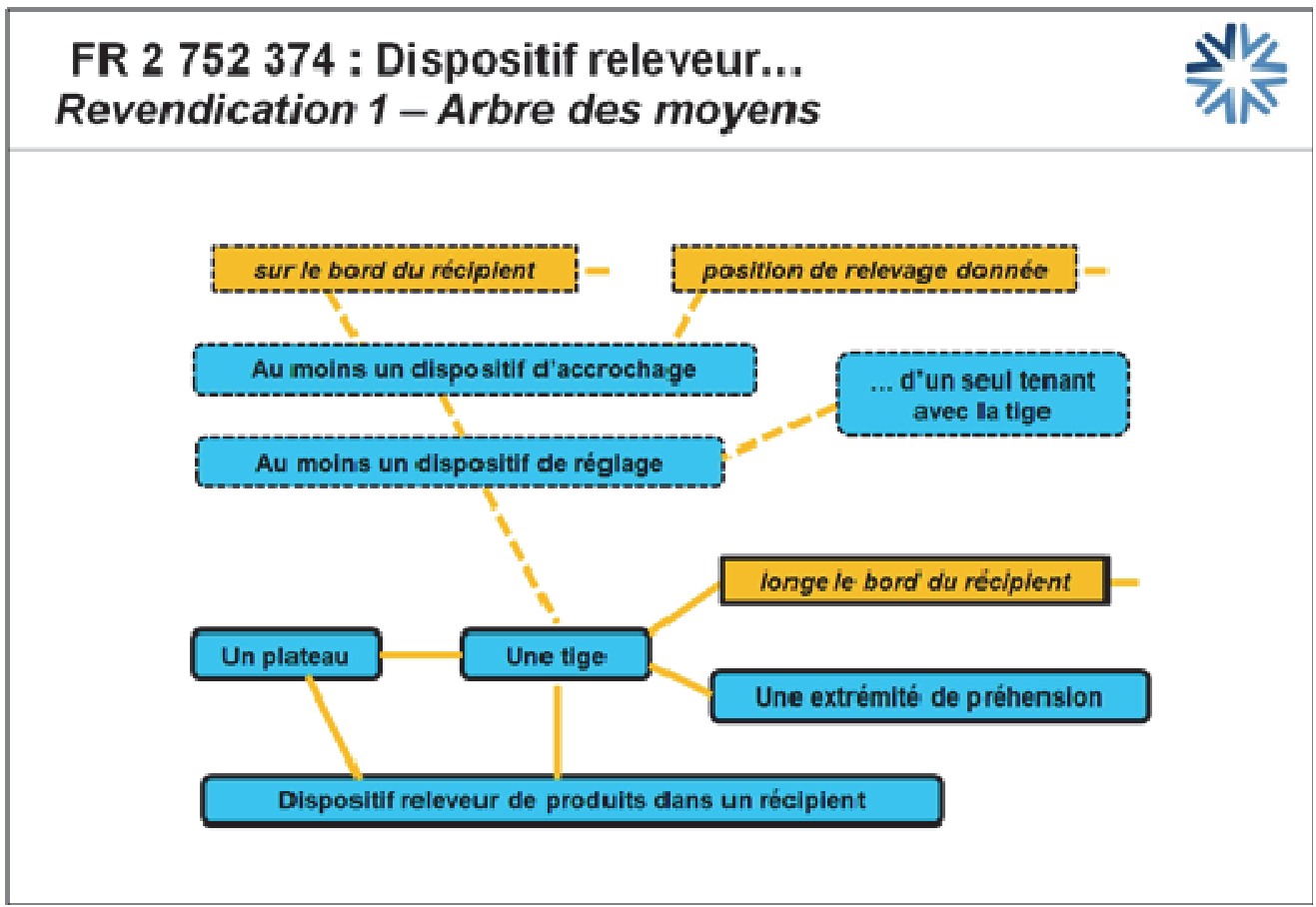

Figure 4. Représentation de la revendication 1 avec l'arbre des moyens - Source : auteur

Les éléments utilisés pour la construction d'un arbre des moyens sont les suivants :

- Rectangle bleu pour un moyen ;

- Lien orange pour une relation « indépendante du temps »;

- Flèche orange pour une relation « dépendante du temps » (procédé) ; 
- Rectangle orange sur le lien ou la flèche lorsque la relation est décrite ;

- « Trait plein » pour un moyen ou une relation de la solution existante « de référence »;

- « Trait pointillé » pour un moyen ou une relation qui sont nouveaux par rapport à la solution existante « de référence ».

Grâce à cet arbre des moyens, on comprend facilement, pour l'exemple présenté :

- qu'il existait déjà des dispositifs releveurs, comprenant un plateau et une tige qui longe le bord du récipient ;

- que l'invention porte sur le fait de mettre des crochets sur la tige pour l'accrocher sur le bord du récipient.

L'expérience montre que la plupart des concepteurs sont totalement «bloqués » devant une revendication sous sa forme classique. En revanche, ils s'approprient rapidement un arbre des moyens, discutent des combinaisons de moyens présentées, proposent des variantes...

C'est logique : les concepteurs sont habitués à raisonner sur des schémas !

Ainsi, grâce à l'arbre des moyens, le langage des brevets peut devenir un langage commun de « conception-protection » pour tous les innovateurs.

\subsection{L'arbre des moyens : des utilisations variées}

Depuis quelques mois, l'utilisation de l'arbre des moyens se développe rapidement dans les entreprises sous des formes variées :

- Formations à l'utilisation des brevets et de la propriété intellectuelle (PI) ;

- Utilisations des brevets existants (veille brevets, extension des brevets...);

- Séances de créativité brevets ;

- Préparation des dépôts de brevets ;

\subsubsection{Formation à l'utilisation des brevets et de la propriété intellectuelle (PI)}

La plupart des entreprises qui innovent dans le domaine technique ont mis en place des formations pour que des acteurs variés, en particulier les concepteurs, sachent utiliser les brevets et les règles du jeu de la PI.

L'arbre des moyens permet de dynamiser ces formations. En effet, il permet d'enrichir les séquences consacrées à :

- La lecture des brevets;

- La créativité brevets ;

- La rédaction des projets de brevets ;

- La coopération entre les concepteurs et les spécialistes PI.

\subsubsection{Utilisation des brevets existants}

L'arbre des moyens permet à des acteurs variés (décideurs, concepteurs...) de mieux comprendre ce qui est protégé par chaque brevet. Cette compréhension est très utile :

- Pour bien exploiter la veille et les états de l'art brevets :

- analyse des brevets ; 
- réunions pour exploiter les brevets sélectionnés ;

- Pour développer l'expertise technique (animée par les référents techniques) :

- Connaissance de l'état de l'art ;

- Connaissance des solutions protégées par l'entreprise et par les tiers ;

- Connaissance de la traçabilité des choix de conception ;

- Pour éclairer les décisions qui sont prises, en intégrant bien les trois regards (stratégie - technique - juridique) pour :

- Le cadrage des projets d'innovation ;

- La mise en place des projets de coopération ;

- Les études de liberté d'exploitation ;

- Les dépôts, extensions et abandons des brevets ;

- L'étude des contrefaçons potentielles ;

- L'exploitation des brevets ;

\subsubsection{Séances de créativité brevets}

L'arbre des moyens fait apparaitre les combinaisons de moyens qui sont au cœur des solutions techniques, qu'elles soient brevetées ou non.

En partant de ces combinaisons de moyens, il est facile de faire de la créativité par :

- Substitution : en remplaçant un moyen par un autre moyen, une relation entre des moyens par une autre relation...;

- Transfert : en identifiant des moyens et des relations, qui sont utilisés dans d'autres domaines techniques, et en essayant de les transférer dans son domaine technique.

Par ailleurs, l'arbre des moyens peut être utilisé pour formaliser et « challenger » les idées de solutions qui sont générées par toutes les méthodes de créativité (brain storming, TRIZ...).

\subsubsection{Préparation des dépôts de brevets}

L'arbre des moyens permet aux concepteurs et aux spécialistes des brevets (Ingénieurs brevets, Conseils en propriété industrielle...) de mieux se comprendre. L'arbre des moyens apporte donc aux entreprises qui l'utilisent des gains importants :

- Amélioration de la qualité des brevets déposés ;

- Gain de temps pour les concepteurs et les spécialistes des brevets (donc réduction des coûts) ;

- Réduction des délais de préparation des dépôts de brevets ;

- Amélioration de la qualité de la relation entre les concepteurs et les spécialistes des brevets, ce qui est apprécié par tous.

\subsection{L'arbre des moyens : un " turbo " pour l'utilisation de la démarche "Innover grâce aux brevets "}

Toutes les utilisations de l'arbre des moyens s'inscrivent dans une démarche globale appelée « Innover grâce aux brevets ». Cette démarche part du principe que les brevets ne sont pas seulement un « résultat juridique » des projets d'innovation, mais que les brevets sont utiles pour toutes les étapes de l'innovation. 
En effet, les brevets sont à la fois :

- Un outil stratégique pour protéger et exploiter les innovations ;

- Une mine d'informations ;

- Un langage de conception puissant.

Grâce à ces trois fonctions complémentaires, les brevets permettent d'éclairer, dynamiser, sécuriser et mieux exploiter les projets d'innovation.

Les deux schémas qui suivent présentent :

- Les principaux bénéfices qui sont liés à l'utilisation des brevets ;

- Les activités brevets qui permettent de générer ces bénéfices.

Dans ces deux schémas, les bénéfices et les activités sont positionnés par rapport aux étapes du processus d'innovation. La taille des intitulés reflète leur importance pour une entreprise qui a été prise comme modèle. Dans chaque entreprise, l'importance des bénéfices et des activités dépend, bien entendu, des choix effectués par ses dirigeants.

La Figure 5 distingue deux types de bénéfices : les bénéfices qui sont liés aux dépôts de brevets (intitulés encadrés) et les bénéfices qui sont liés à l'utilisation « gratuite» des brevets.

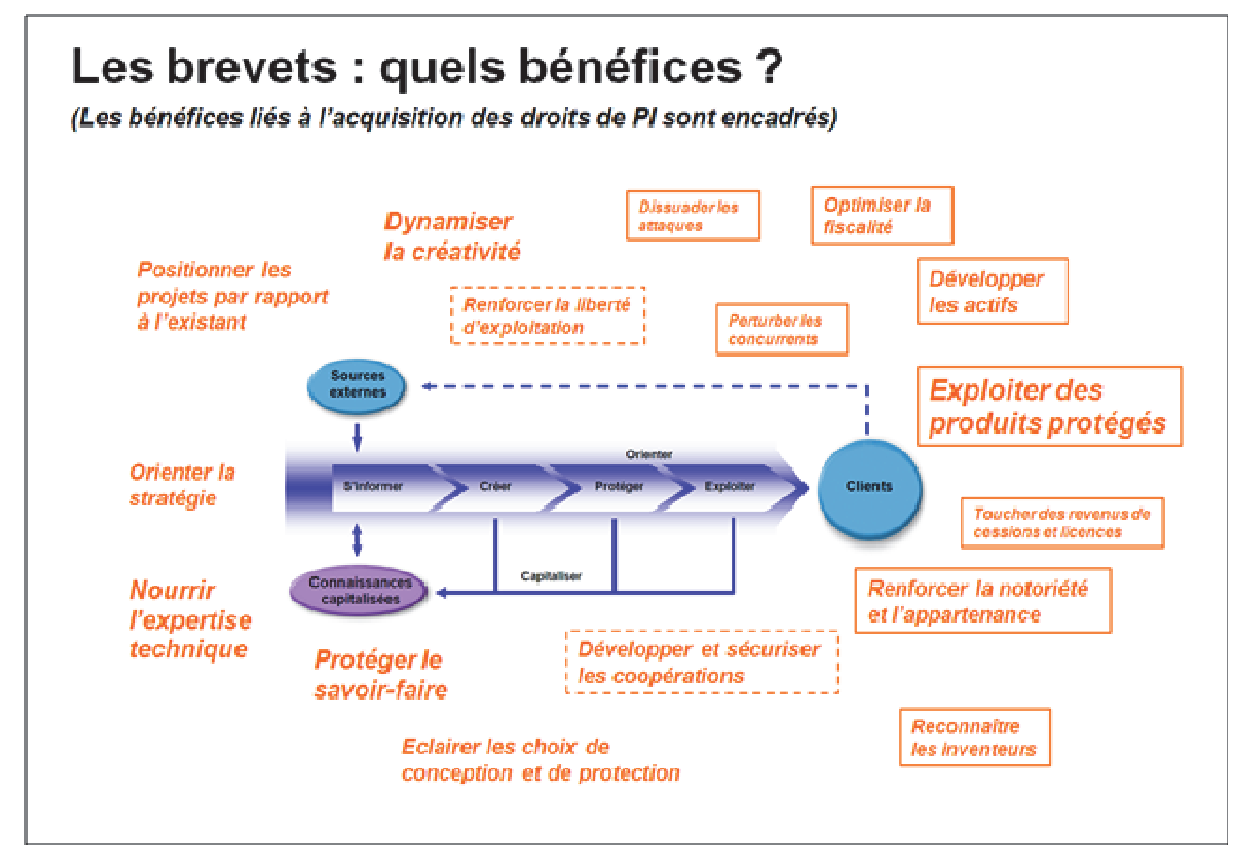

Figure 5. Les bénéfices des brevets - Source : auteur

La Figure 6 montre que les «activités brevets » sont nombreuses et souvent liées entre elles. L'utilisation des brevets est donc à la fois riche et complexe. Les bénéfices obtenus par les entreprises dépendent donc directement de la qualité des coopérations entre les spécialistes des brevets (internes à l'entreprise et/ou externes) et les non-spécialistes.

D'où l'intérêt de l'arbre des moyens : un langage commun, dont l'utilisation est simple.

L'expérience montre que toutes les personnes qui utilisent les brevets s'approprient rapidement l'arbre des moyens, ce qui a conduit le responsable PI d'une grande entreprise à déclarer : "Ce petit outil n'a l'air de rien, mais il pourrait changer pas mal de choses dans le monde des brevets! ». 


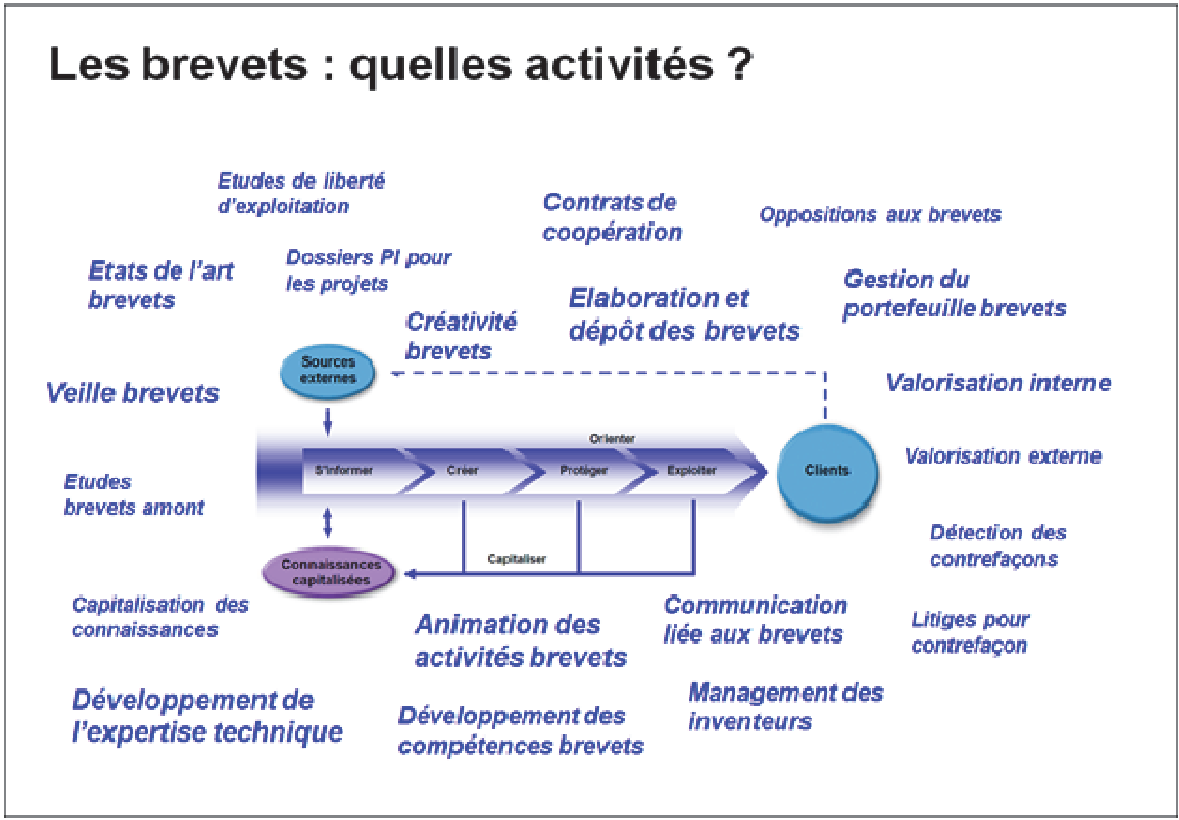

Figure 6. Les activités liées aux brevets - (Source : auteur)

De plus, au-delà des nombreuses utilisations directes des brevets qui viennent d'être présentées, le système des brevets peut servir de modèle pour dynamiser le management des connaissances pour l'innovation.

\section{Le système des brevets : un modèle pour le management des connaissances pour l'innovation}

Comme cela a été présenté précédemment (\$ 3.2), le système des brevets a été créé pour accélérer le progrès technique, grâce à une reconnaissance pertinente des créateurs et un bon partage des connaissances.

Cette finalité est très proche de ce que recherchent, de plus en plus, toutes les entreprises : motiver les innovateurs et mieux partager les connaissances pour mieux innover (Ermine 2003), (Saulais 2013).

Depuis plus de deux siècles, le système des brevets a fait la preuve de son efficacité dans ce domaine. Des millions d'innovateurs, qui travaillent dans des entreprises variées, partout dans le monde, ont accès gratuitement et facilement à plus de 80 millions de documents. Ces documents sont très riches : ils mémorisent l'histoire des inventions depuis plus de deux siècles, dans tous les domaines techniques.

Les pères du système des brevets, en particulier Benjamin Franklin et Thomas Jefferson (deux inventeurs qui ont participé à la rédaction de la Constitution Américaine, après la guerre d'indépendance) ne pouvaient pas imaginer, en 1790, la révolution qu'allait apporter l'informatique à la fin du $20^{\mathrm{e}}$ siècle. Et pourtant, quand le système des brevets a été informatisé, rien n'a été changé dans la structuration des connaissances de ce système (structuration des brevets, structuration des bases brevets, procédures). La mine d'or de la base mondiale des brevets existait depuis des dizaines d'années. Maintenant, grâce à l'informatique tous les innovateurs peuvent exploiter cette mine d'or.

Par ailleurs, depuis 1790, il n'a jamais été nécessaire de « faire le ménage » dans les 80 millions de documents de la base mondiale des brevets. Quelle est l'entreprise qui peut en dire autant pour son intranet dédié à l'innovation (beaucoup plus récent et comportant beaucoup moins de documents !) ? Quel est le secret de cette performance ? 
Ce secret est simple, et toutes les entreprises peuvent s'en inspirer. La structuration des brevets focalise ces documents sur l'essentiel : la traçabilité des choix de conception. Ainsi chaque brevet, au moment de son dépôt, fait un état de l'art et propose une solution technique nouvelle qui améliore l'existant. Un brevet ne gêne donc pas les brevets qui le suivront. Au contraire : c'est un maillon dans l'histoire des techniques.

Cette structuration du brevet est donc très efficace pour capitaliser les connaissances, dans le but de dynamiser l'innovation :

- La description d'un brevet correspond à la liste des questions qu'il faut se poser pour innover ;

- Les revendications d'un brevet vont au cœur de la conception en posant la question clé : "Quelles sont les combinaisons nouvelles de moyens proposées?»

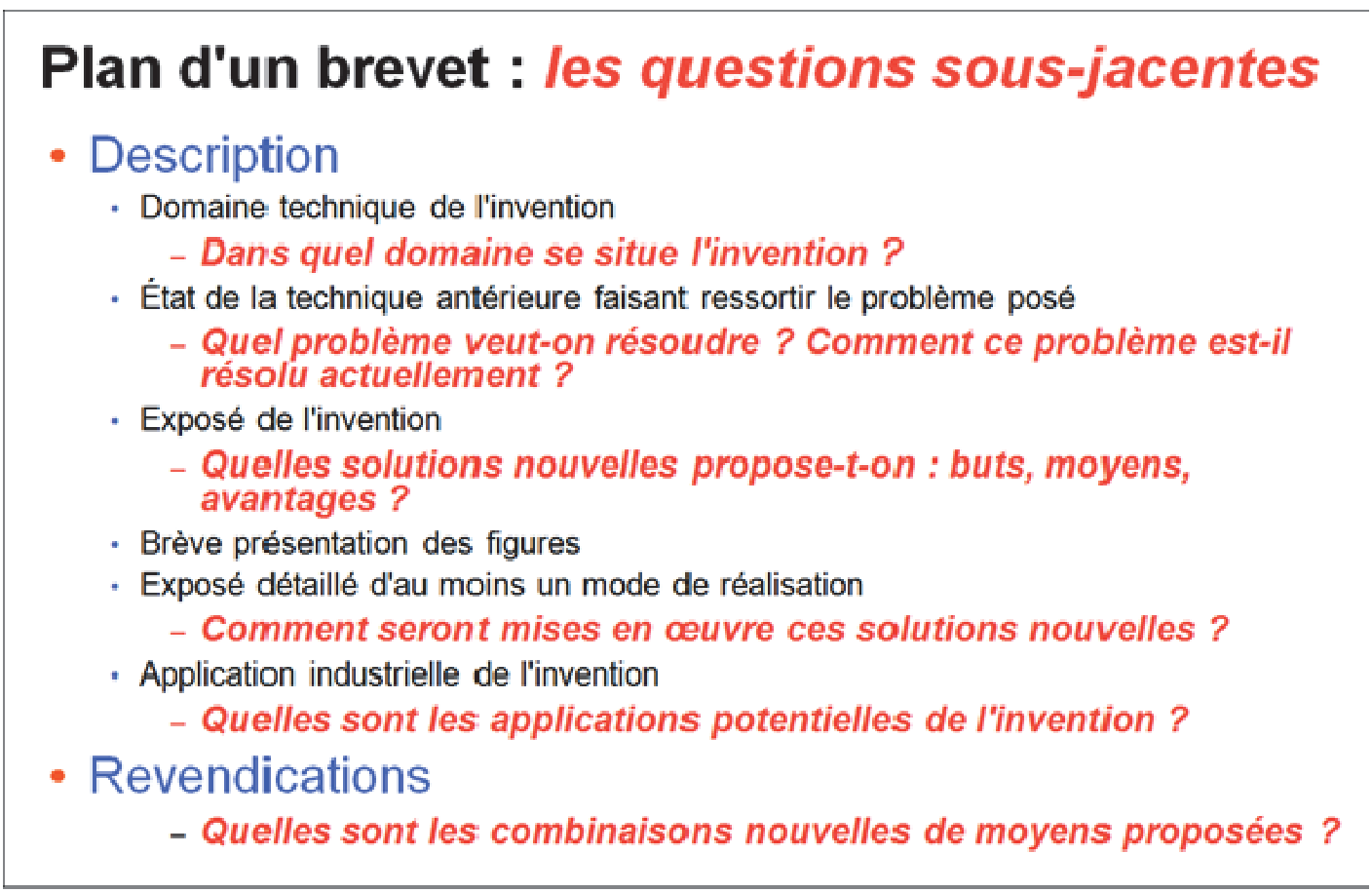

Figure 7. Les questions sous-jacentes au plan du brevet - Source : auteur

Cette structuration remarquable des brevets, présentée sur la

Figure 7, a donné naissance à deux formulaires très utiles pour manager l'innovation... et les innovateurs : le «mémo-innovation» et la «fiche-connaissances». Ces deux formulaires sont des outils simples, puissants et universels. Les entreprises les utilisent pour tous les types d'innovations (produits techniques, services proposés, organisation interne...) et chaque personne peut également les utiliser pour sa vie personnelle (pour améliorer le rangement de sa cave, mieux s'entendre avec son voisin de palier, développer sa maîtrise de l'anglais...).

Cela marche vraiment pour tous les sujets !

Le mémo-innovation présenté en Figure 8, inspiré du brevet, reprend et adapte, en première page, les questions qu'il faut se poser pour rédiger un brevet. Les questions de la deuxième page prolongent la logique des brevets et permettent, au sein d'une entreprise, de partager davantage de connaissances, en particulier pour les projets d'innovation, avec des questions comme :

- Quelles sont les limites/défauts des solutions retenues?

- Quelles autres solutions ont été imaginées/étudiées ? Pourquoi n'ont-elles pas été retenues ? 


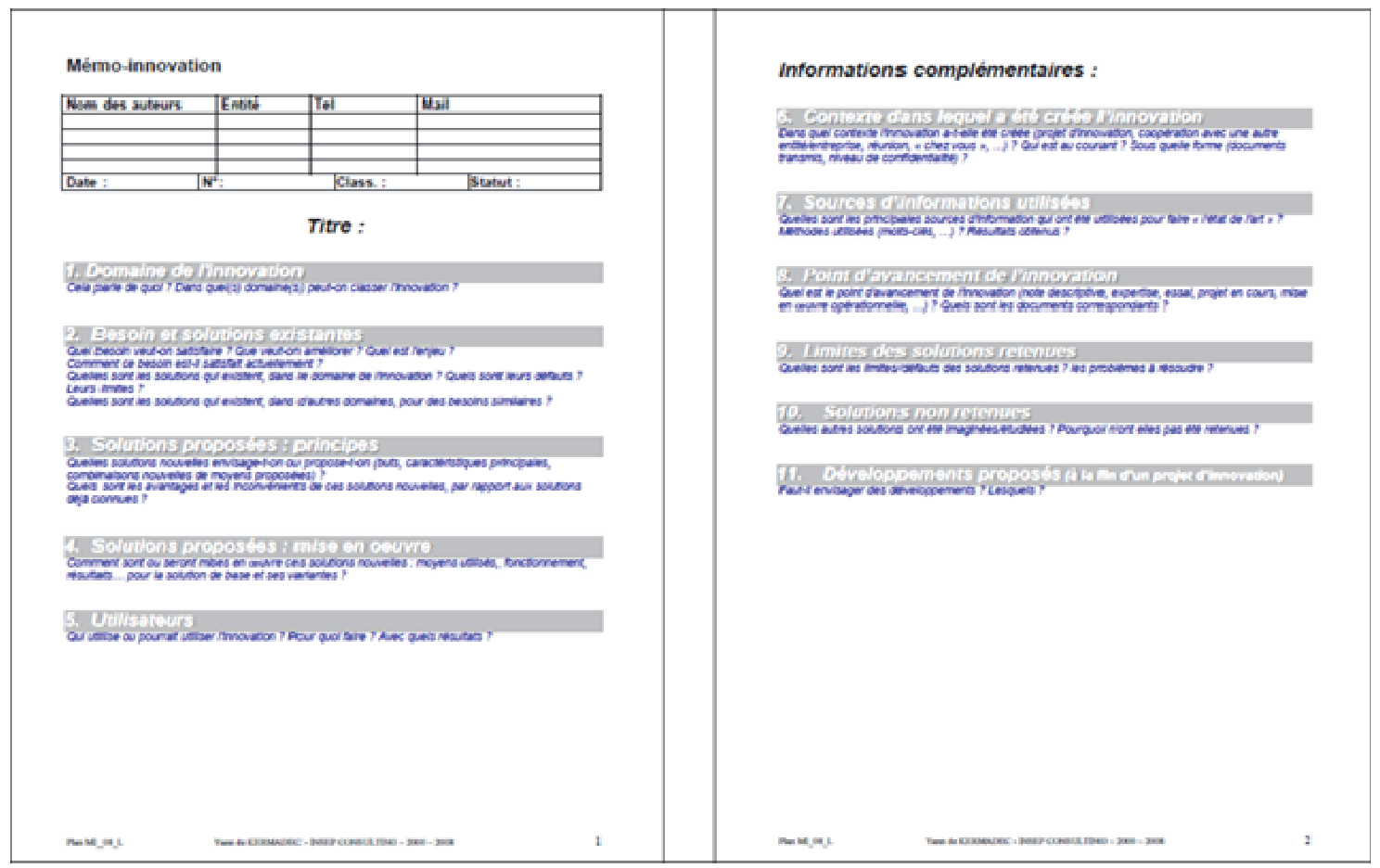

Figure 8. Le mémo-innovation - Source : auteur

La fiche-connaissances présentée en Figure 9, inspirée du résumé du brevet (la première page du brevet) est une sorte « d'affiche de pub » du module de connaissances correspondant. La fiche-connaissances a, comme le résumé du brevet, deux fonctions principales :

- Présenter le module de connaissances (mémo-innovations, expertises, supports de formation, notices de dépannage de machines, présentation de sites internet...) de manière synthétique ;

- Faciliter la recherche des modules de connaissances pertinents et faciliter la prise de contact avec les personnes compétentes sur un sujet.

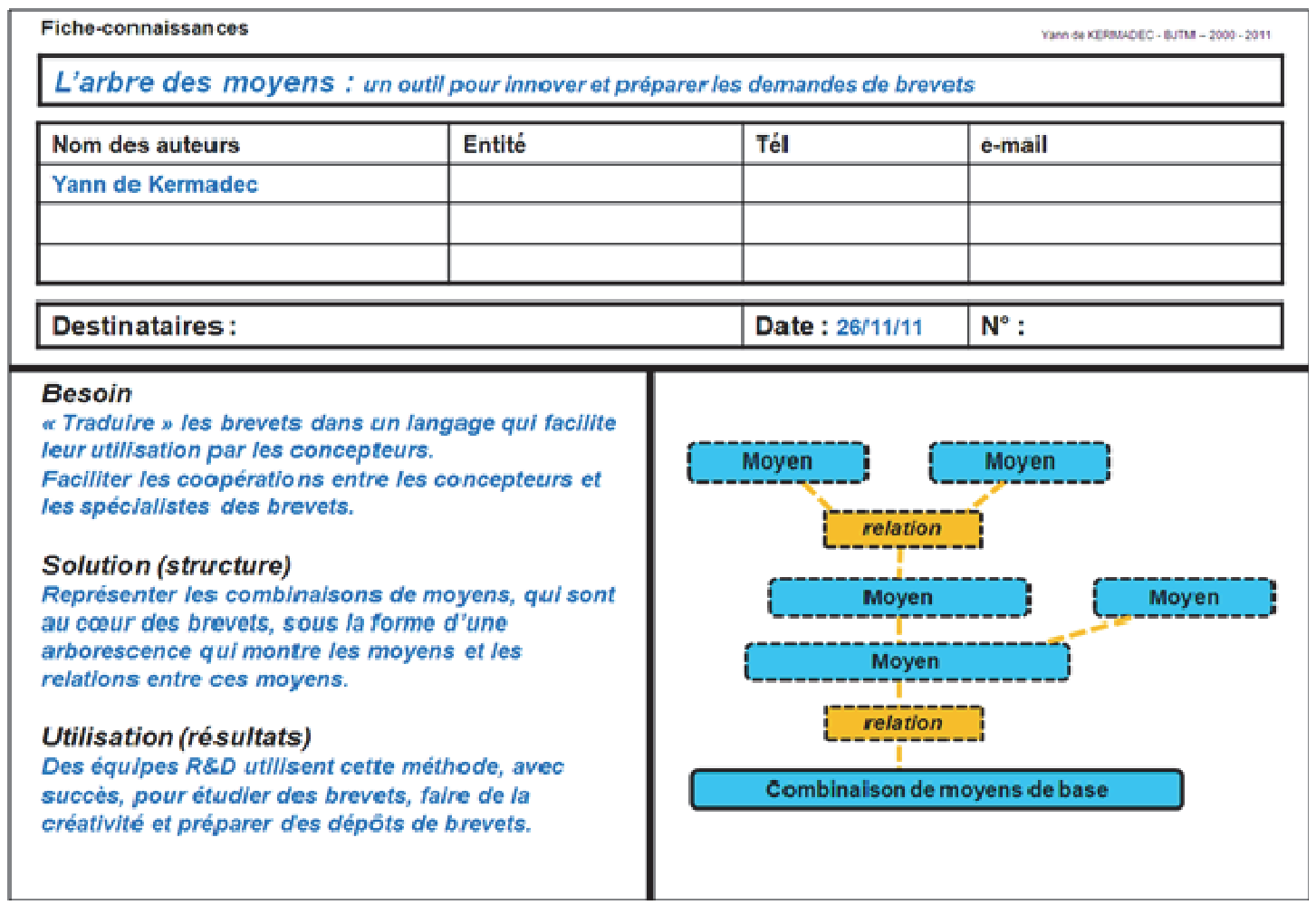

Figure 9. La fiche-connaissances - Source : auteur 
Cet exemple de fiche-connaissances qui présente «l'arbre des moyens » nous permet, pour terminer, de revenir à une innovation qui est au cœur de cet article.

En effet, l'arbre des moyens est le langage qui permet de bien utiliser le brevet, le « couteau suisse » des innovateurs... qui est très efficace pour coopérer, inventer et innover.

\section{Conclusion}

Cet article met bien en évidence le fait que les brevets sont à la fois :

- un outil stratégique pour protéger et exploiter les innovations ;

- une mine d'informations ;

- un langage de conception puissant.

Grâce à ces trois fonctions complémentaires, les brevets permettent d'éclairer, dynamiser, sécuriser et mieux exploiter les projets d'innovation. Avec des utilisations aussi variées, le brevet est véritablement un « couteau suisse » au service des innovateurs !

Le système des brevets existe depuis plus de deux siècles. Pourtant, le principe de base de ce système est plus que jamais d'actualité : dynamiser l'innovation avec un bon partage des connaissances et une protection efficace des entreprises qui innovent.

Au fil du temps, la structuration des connaissances dans les brevets et dans les bases brevets a évolué pour répondre aux exigences croissantes des états et des entreprises. Cette structuration des connaissances est logique et puissante. Le système des brevets peut être considéré, aujourd'hui, comme un modèle de management des connaissances pour l'innovation.

Si le système des brevets n'est pas miné par une dérive juridico-financière, c'est dans les années qui viennent, avec "l'innovation ouverte», que le système des brevets va donner sa pleine mesure. En effet, sans une vision claire de « qui est propriétaire de quoi », et de « qui sera propriétaire de quoi », il n'est pas possible d'avoir des coopérations efficaces et paisibles.

Par ailleurs, la richesse de l'information technique, en accès libre dans les bases brevets, est encore sous exploitée. Dès que cette mine d'or sera bien exploitée, le centre de gravité de l'utilisation des brevets se positionnera, de manière saine et naturelle, au cœur de la conception et de l'innovation.

Mais pour cela, il faut que les concepteurs aiment les brevets, donc comprennent le langage des brevets et la puissance de ce langage pour la conception. L'arbre des moyens, qui est présenté dans cet article, pourrait fortement y contribuer, en particulier si les jeunes qui font des études supérieures techniques se l'approprient et l'utilisent pendant leurs études.

En effet, si des étudiants utilisent l'arbre des moyens dans le cadre de leurs stages en entreprise, pour leurs travaux de fin d'études et pour leurs thèses, ils seront rapidement passionnés par les brevets... et transmettront cette passion, et les compétences associées, aux personnes avec lesquelles ils travailleront dans les entreprises.

\section{Références bibliographiques}

ALTER N., L'innovation ordinaire. Eyrolles, 2000.

BEFFA K., VILLANI C., Les coulisses de la création. Flammarion, 2015.

BREESÉ, P., Straégies de propriété industrielle. Dunod, 2002.

CCI-PARIS, Beta-Strasbourg, L'innovation ouverte - Définition, pratiques et perspectives. Chambre de commerce et d'industrie de Paris, 2013. 
COllectif-Robert., Le Dictionnaire historique de la langue Française. Le Robert, 2012.

DRUCKER P., HESSELBEIN F., Les cinq questions fondamentales du management. Diateino, 2015.

ERMINE J.-L., La gestion des connaissances. Hermès-Lavoisier, 2003.

FONTANET X., Si on faisait confiance aux entrepreneurs . Les Belles Lettres, 2010.

GENELOT D., Manager dans la complexité. 3e. INSEP Editions, 2001.

GoMEZ P.-Y., Le travail invisible. François Bourin Editeur, 2013.

KERMADEC DE Y., Innover grâce aux brevets. 2e. INSEP Editions, 2001.

KERMADEC DE Y., Innover, c'est l'affaire de tous. 2e. INSEP Editions, 2009.

KERMADEC DE Y., BREESÉ P., La propriété intellectuelle au service de l'innovation. 4e. Nathan, 2015.

KERMADEC DE Y., PME : pensez propriété intellectuelle ! DGCIS, 2010.

NOYE D., Réussir les changements difficiles. INSEP Editions, 2002.

PMI, Management de projet - un référentiel de connaissances. AFNOR, 2000.

RADJOU N., L'innovation frugale. Diateino, 2015.

RouXEL J., Les Shadocks. Editions Circonflexe, 2003.

SAULAIS P., «Application de la gestion des connaissances à la créativité des experts et à la planification de la R\&T en milieu industriel de haute technologie » Thèse de doctorat, Télécom Ecole de Management, 2013. 